\title{
Otoacoustic Emissions and Evoked Potentials in Infants after Breast-Feeding Jaundice
}

\author{
-Hearing Dysfunction in Breast-Feeding Jaundice
}

\author{
Adrián Poblano $^{1 *}$, Norma Ballesteros ${ }^{1}$, Carmina Arteaga ${ }^{1}$, Blanca Flores ${ }^{2}$, Teodoro Flores ${ }^{2}$ \\ ${ }^{1}$ Laboratory of Cognitive Neurophysiology, National Institute of Rehabilitation, Mexico City, Mexico; ${ }^{2}$ Laboratory of Clinical Neu- \\ rophysiology, National Institute of Rehabilitation, Mexico City, Mexico. \\ Email: *drdeaf@starmedia.com
}

Received November $11^{\text {th }}, 2011$; revised May $28^{\text {th }}, 2012$; accepted June $29^{\text {th }}, 2012$

\begin{abstract}
We study hearing in a group of infants with Breast-feeding jaundice (BFJ) by means of Transient-evoked otoacoustic emissions (T-EOE) and Brainstem auditory evoked potentials (BAEP) searching for relationship between bilirubin serum levels and auditory dysfunction. Eleven infants born at-term with BFJ were selected for the study. We studied also 11 control age- and gender matched healthy at-term infants without signs of jaundice. T-EOAE studies were performed between 5 - 7 days after birth, and 3 months later. BAEP studies were performed once. BFJ group infants exhibited lower amplitudes in T-EOE than infants in the control group. These differences disappear at the 3-month evaluation. In BAEP, we observed a significant latency delay of waves I and V in Breast-feeding jaundice group infants. All infants in both groups demonstrated reproducible wave $\mathrm{V}$ response at 30 decibels. No significant correlation values were observed between bilirubin serum levels and T-EOE and BAEP variables. Our data suggest that BFJ can result in transient peripheral and central auditory dysfunction. Dysfunction is reversible after treatment of infants with BFJ.
\end{abstract}

Keywords: Breast-Feed Jaundice; Hyperbilirubinemia; Hearing; Neonates; Transient-Evoked Otoacoustic Emissions; Brainstem Auditory Evoked Potentials

\section{Introduction}

Previous research has shown the relationship between neonatal hyperbilirubinemia and sensorineural hearing loss $[1,2]$. The majority of investigations have been performed by means of Brainstem auditory evoked responses (BAEP) in High-risk newborns (HR-NB). These infants have been followed throughout several years after birth to confirm hearing loss [3-6]. Observations have suggested repeatedly that hearing dysfunction is a manifestation of injury situated in the auditory neural pathway along the brainstem. However, one paper had situated the injury also in the peripheral cochlear system [7].

Breast-feeding jaundice (BFJ) provides a fair model to study the peripheral and central auditory alterations after pure neonatal hyperbilirubinemia, because this is different from hyperbilirubinemia in HR-NB. The majority of infants with BFJ are born at term with adequate weight and without other risk factors for auditory damage, such as asphyxia, intracranial hemorrhage, or others. Thus, the aim of our study was to study hearing function in a group of infants with BFJ by means of Transient-evoked oto-

${ }^{*}$ Corresponding author. acoustic emissions (T-EOE) and BAEP with the hypothesis that there is a relationship between bilirubin serum levels and peripheral and central auditory dysfunction. In order to test our hypothesis, we employed T-EOE as an index for cochlear toxicity, and BAEP as an index of auditory brainstem neurotoxicity.

\section{Materials and Methods}

\subsection{Subjects}

Healthy infants born at General Hospital "Dr. Darío Fernández" readmitted with jaundice to the Department of Pediatrics during their first week of life with diagnoses of BFJ were selected for the study. Inclusion criteria included the following: jaundice in Kramer zone $\geq 2$; bilirubin levels $>15 \mathrm{mg} / \mathrm{dL}$; age at birth between 37 and 41 of gestational weeks; weight $>2750 \mathrm{~g}$; 5-min Apgar score $>7$, and without other risk factors for hearing damage. All of the infants were exclusively breastfed. Mothers of the patients noted jaundice at home, and it was not accompanied by decreased activity or poor feeding. None newborn had any laboratory evidence of hemolytic disease as evidenced by anemia, reticulocyto- 
sis, or another abnormality. Infants were screened for neurological, and auditory dysfunction [8,9], and for perinatal factors associated with an increased risk of hyperbilirubinemia including, maternal diabetes mellitus, high blood pressure, administration of oxytocin during labor, as well as central nervous system bleeding or injury, or infection. At time of readmission to the hospital, the infants were evaluated for clinical signs of acute bilirubin encephalopathy manifested as changes in level of consciousness, tone/movement, poor feeding, abnormal cry, opisthotonus and/or seizures, and alterations in brainstem function. Other causes of hyperbilirubinemia including scalp bruising, dehydration and sepsis were investigated. Laboratory studies included complete blood counts, electrolytes, glucose, and cultures. Cranial Ultrasonographic studies were performed in all newborns to rule-out other brain pathologies. Bilirubin determinations and T-EOE were performed in the first days after jaundice was recognized to identify and treat possible neurodevelopment deviations; T-EOE were repeated 3 months later. BAEP determinations were performed rapidly after T-EOE examination once. We studied 11 infants with BFJ (four males and seven females) and 11 control healthy newborn infants who were age- and gender matched at the time of study without signs of jaundice. Results of each study were blinded to the researchers who revised BAEP and T-EOE determinations for group pertinence. The Research and Ethics Committees of the Hospital and of the Institute approved the protocol. Informed consent was obtained from parents of infants and is contained in signed forms according to the principles expressed in the Declaration of Helsinki.

\subsection{Bilirubin Determinations}

Samples were obtained by peripheral venipuncture. All specimens were protected from light after they were drawn, and these were analyzed immediately. Total bilirubin concentration was determined spectrophotometrically, and unbound bilirubin concentration was measured by the peroxidase method using a clinical analyzer (UB analyzer, Arrows Co., Osaka, Japan). Samples with high unbound bilirubin $>0.5 \mu \mathrm{g} / \mathrm{dL}$ were measured for concentration of direct bilirubin by diazo method [10].

\subsection{Transient-Evoked Otoacoustic Emissions (T-EOE)}

Responses were recorded with the ILO-88 (Otodynamic Ltd, London, UK), during a sleep session. Studies were conducted in a sound-proofed room. Prior to the Otoacoustic emission study, ear canals were checked with an otoscope to confirm permeability of external auditory channel. We use a miniature acoustic probe fitted into the infant ear channel. The probe incorporates a small micro- phone and ear-speaker. Stimuli consisted of 80 decibel (dB) HL (Hearing level) click stimulation of $100 \mu \mathrm{sec}$ delivered at a repetition rate of $11 / \mathrm{sec}$. Signal was amplified and filtered between 350 and $6400 \mathrm{~Hz}$. Two hundred fifty stimuli were averaged. Analysis time was 20 millisec. Contralateral ear masking with white noise $20 \mathrm{~dB}$ below stimulus intensity was administrated simultaneously. Adequate T-EOAE responses were identified based on reproducibility of $\geq 75 \%$. The detection threshold of T-EOAE was measured by stimulating the ear with decreasing click intensities in $20-\mathrm{dB}$ steps. Each ear was tested separately [11].

\subsection{Brainstem Auditory Evoked Potentials (BAEP)}

BAEP were recorded and analyzed following standard international recommendations [12]. BAEP determinations were performed after a feeding sleep in a soundproof room. Each subject was tested with BAEP using an ATI system (Buenos Aires, Argentina). Three gold-disk electrodes were placed on the scalp, with negative electrode on ipsilateral mastoid, positive on vertex, and neutral on contralateral mastoid. Interelectrode impedances were $\leq 2$ kilo-ohms. Electrical activity among electrodes was amplified and averaged over a 10 millisec time base. Stimulus was administrated through a Telephonics TDH49 earphone (Telephonics Co., Huntington, NY, USA). Stimuli were presented monoaurally at a rate of $11 / \mathrm{sec}$. Initial presentation intensity was $70-\mathrm{dB}$ HL and decreased by $20-\mathrm{dB}$ steps to determine neurophysiologic threshold of response. Contralateral ear masking with white noise $20 \mathrm{~dB}$ below stimulus intensity was administrated simultaneously. Stimuli were delivered mono-aurally and consisted of $100 \mu \mathrm{sec}$ alternating clicks. Band pass filters were set between 100 and $3000 \mathrm{~Hz}$, and stimulus average was 2024 clicks. The process was repeated at least once to ensure reproducibility of response. Latencies of waves I, III, and V were measured by manual cursor placement at left and right ear recordings separately; I-III, III-V, and I-V interpeak intervals were calculated automatically by computer software; a normal hearing threshold was determined if the reproducible wave $\mathrm{V}$ response was present at last at $30 \mathrm{~dB}$ level.

\subsection{Data Analysis}

We calculate average and Standard deviation (SD) of quantitative variables and percentages of qualitative variables. We report BAEP data from the ear producing the faster I-V conduction time as published previously elsewhere $[13,14]$, because in this way we avoid subclinical hearing conductive alterations. Comparisons between both groups were performed by two-tailed Student 
$t$ test. We performed Pearson correlation analysis between total, indirect, and direct bilirubin serum levels and T-EOE and BAEP variables. The level of statistical significance a-priori chosen was $p \leq 0.05$. Calculations were performed with the SPSS 14.0 (SPSS Inc., Chicago, IL, USA) computer program [15].

\section{Results}

Clinical characteristics of infants in each group are shown in Table 1; no significant differences in compared variables between groups were observed. The infants age at which mother requested attention ranged between 5 and 7 days after birth (median, 6 days). Average bilirubin determinations in BFJ-group infants are presented in Table 2.

Averages of response amplitude by ear determined by T-EOE at birth and 3 months later are shown in Table 3; in the neonatal period, there were significant differences in both ear responses between groups, with BFJ-group infants exhibiting lower amplitudes than infants in the control group. However, these differences disappear at the 3-month evaluation when hyperbilirubinemia was resolved.

Average of main waves (I, III, and V) and interwave intervals (I-III, III-V, and I-V) in BAEP determinations are presented in Table 4; we observed a significant latency delay of waves $\mathrm{I}$ and $\mathrm{V}$ in BFJ-group infants. However, all infants in both groups demonstrated reproducible wave $\mathrm{V}$ response at $30 \mathrm{~dB}$, suggesting adequate hearing and thus no second BAEP study was performed in infants. We did not observe cases of auditory neuropathy. No significant correlation values were observed between total, indirect, and direct bilirubin serum levels and T-EOE, and BAEP variables.

Table 1. Clinical characteristics of control $(n=11)$ and infants with Breast-feeding jaundice (BFJ) $(n=11)$.

\begin{tabular}{ccc}
\hline & Control $(\mathrm{x} \pm \mathrm{SD})$ & $\mathrm{BFJ}(\mathrm{x} \pm \mathrm{SD})$ \\
\hline Weight at birth $(\mathrm{g})$ & $2897 \pm 371$ & $2863 \pm 350$ \\
Age at birth (weeks) & $37.9 \pm 1.23$ & $38.3 \pm 0.84$ \\
1 min Apgar score & $7.6 \pm 1.21$ & $8.0 \pm 0.52$ \\
5 min Apgar score & $9 \pm 0$ & $9 \pm 0$ \\
\hline
\end{tabular}

$\mathrm{x}=$ average $\mathrm{SD}=$ Standard deviation.

Table 2. Average of maximum bilirubin serum levels $(\mathrm{mg} / \mathrm{dL})$ in infants with Breast-feeding jaundice (BFJ).

\begin{tabular}{rcc}
\hline & $\mathrm{x}$ & $\mathrm{SD}$ \\
\hline Total bilirubin & 18.00 & 0.64 \\
Direct bilirubin & 0.65 & 0.12 \\
Indirect bilirubin & 17.35 & 0.60 \\
\hline
\end{tabular}

$\mathrm{x}=$ average $\mathrm{SD}=$ Standard deviation.
Table 3. Average \pm standard deviation of amplitude responses (dB) in T-EOE in control and BFJ-group infants.

\begin{tabular}{ccccc}
\hline & $\begin{array}{c}\text { Right ear } \\
1 \text { week }\end{array}$ & $\begin{array}{c}\text { Left ear } \\
1 \text { week }\end{array}$ & $\begin{array}{c}\text { Right ear } \\
3 \mathrm{~m}\end{array}$ & $\begin{array}{c}\text { Left ear } \\
3 \mathrm{~m}\end{array}$ \\
\hline $\begin{array}{c}\text { Control } \\
\text { group }\end{array}$ & $17.96 \pm 7.93$ & $19.08 \pm 9.38$ & $16.10 \pm 6.02$ & $18.60 \pm 7.01$ \\
$\begin{array}{c}\text { BFJ } \\
\text { group } \\
p\end{array}$ & $12.48 \pm 8.74$ & $13.02 \pm 7.57$ & $16.60 \pm 8.00$ & $19.14 \pm 6.25$ \\
$p$ & 0.05 & 0.04 & $\mathrm{~ns}$ & $\mathrm{~ns}$ \\
\hline
\end{tabular}

T-EOE $=$ Transient-Evoked otoacoustic emissions; BFJ = Breast feeding jaundice; not significant.

Table 4. Average \pm standard deviation of latencies (msec) of main waves and interval interwaves of BAEP at $80 \mathrm{~dB}$ in control infants and in infants with BFJ.

\begin{tabular}{cccc}
\hline Wave/Interval $(\mathrm{msec})$ & Controls & BFJ & $\mathrm{p}$ \\
\hline I & $1.70 \pm 0.13$ & $1.83 \pm 0.25$ & 0.04 \\
III & $4.41 \pm 0.18$ & $4.49 \pm 0.23$ & $\mathrm{~ns}$ \\
V & $6.64 \pm 0.26$ & $6.79 \pm 0.29$ & 0.05 \\
I-III & $2.71 \pm 0.14$ & $2.66 \pm 0.21$ & $\mathrm{~ns}$ \\
III-V & $2.23 \pm 0.23$ & $2.30 \pm 0.25$ & $\mathrm{~ns}$ \\
I-V & $4.94 \pm 0.26$ & $4.96 \pm 0.29$ & $\mathrm{~ns}$ \\
\hline
\end{tabular}

$\mathrm{x}=$ average $; \mathrm{SD}=$ standard deviation $; \mathrm{BAEP}=$ Brainstem auditory evoked potentials; $\mathrm{dB}=$ decibels; $\mathrm{BFJ}=$ Breast feeding jaundice; $\mathrm{ns}=$ not significant.

\section{Discussion}

In this paper, we showed that BFJ in low-risk at-term infants results in both auditory peripheral and central dysfunctions as reflected in T-EOE and BAEP alterations. We also observed that these auditory and neurophysiologic alterations could be reversible after rapid management and resolution of the hyperbilirubinemia. However, we could not demonstrate our hypothesis about significant correlation between bilirubin serum levels and peripheral and central auditory dysfunction.

Several in-vitro studies showed that bilirubin-induced neuronal toxicity involves changes in energy metabolism, alteration in membrane function, decreased membrane potential, alteration in enzyme function, and inhibition of protein synthesis $[16,17]$. It also appears from these previously cited in-vitro studies that immature cells are more sensitive to bilirubin toxicity than differentiated cells, supporting clinical experience demonstrating that premature neonates are more susceptible to bilirubininduced neurotoxicity than at-term infants. However, our data also suggest that the auditory responses of otherwise healthy at-term infants could be injured by hyperbilirubinemia.

Various clinical factors, such as hypothermia, hypoxia, acidosis, hypercarbia, asphyxia, sepsis, intraventricular hemorrhage, and hemolysis, have been postulated to ex- 
plain the occurrence of bilirubin neurotoxicity at much lower levels of serum total bilirubin [1]. These factors are thought to increase the risk of kernicterus by affecting serum bilirubin-albumin binding, bilirubin entry into the brain, or tissue uptake of bilirubin. For example, one study identified a degree of acidosis with low serum albumin levels that occurred prior to the maximum unbound bilirubin level and that was present in newborns with kernicterus [18]. On the other hand, a study of follow-up of at-term or near-term infants with BFJ reported transient neurologic abnormalities. These abnormalities resolved following management with phototherapy and exchange transfusions, and bilirubin level did not correlate with the long-term prognosis [19]. Results from this study are partially in agreement with our data.

One of the tools commonly utilized to investigate bilirubin-induced neurotoxicity in neonates is BAEP test. BAEP has been studied in high-risk pre-term newborns as a non-invasive auditory neurophysiologic assessment of bilirubin neurotoxicity. Many observations confirm the higher frequency of auditory alterations in newborns after hyperbilirubinemia, such a delay of wave I, III and $\mathrm{V}$ latencies or the absence of response at higher intensities of auditory stimulation [3-6]. This is in indirect agreement with our observations, because we observed a wave I and V delay. Other studies have shown correlation between indirect bilirubin serum levels and BAEP alterations [20]; however we are unable to confirm this correlation in our observations. Other studies have demonstrated that BAEP alterations are reversible after blood exchange [21]. However, we were unable to confirm this observation, because our infants with BFJ were not blood ex-changed and all our infants in both, control and BFJ groups presented response at $30 \mathrm{~dB}$. Thus, there was no reason for subsequent appointment 3 months later for an additional BAEP study.

Otoacoustic emissions have been used to screen hearing alterations after neonatal hyperbilirubinemia. T-EOE has been employed as a first step in rapid screening of infant auditory performance. Study investigators usually carry out a BAEP examination after an abnormal T-EOE $[2,7]$. Researchers have observed a decrease in the response amplitude of T-EOE such as that which we observed in our BFJ-group infants, or an increased number in absences of response after auditory stimulation.

Recently, Jangaard et al. did not observed an increased frequency in cerebral palsy, developmental delay, autism, and hearing loss in a population of infants born at-term or near-term with other neurologic risk-factors other than hyperbilirubinemia ( $\geq 19 \mathrm{mg} / \mathrm{dL}$ ) [22]. This result is also in indirect agreement with our observations, because we observed no case of hearing loss. However, because we measured T-EOE and BAEP variables quantitatively, we were able to detect subclinical effects of transient hyper- bilirubinemia in the peripheral and central auditory system of infants with BFJ, that were not observed by the other work team.

We did not observe cases of auditory neuropathy in our sample, but this may be explained of our small sample size [23]. An alternative explanation may be that auditory neuropathy after neonatal hyperbilirubinemia is a complex alteration that requires other risk-factors than only high bilirubin serum levels for the production of auditory alterations. However, our infants must be followed, because language delay has been reported as a possible complication after neonatal hyperbilirubinemia [24].

Our research possesses some limitations; thus, our results must be considered as tendencies, not strong conclusions. The sample is small, despite its being highly selected and age-gender-matched to a control group. Therefore, in the future a greater number of at-term infants with BFJ must be studied. Moreover, follow-up time was brief, notwithstanding follow-up of these infants will continue with these as out-patients in the Pediatrics Department to determine whether minimal auditory dysfunction or language alteration during child development can be a result of the BFJ episode during the neonatal period of life.

\section{Conclusion}

Our data suggest that BFJ can result in peripheral and central auditory dysfunction. The dysfunction is reverseble; thus, early identification and treatment of infants with BFJ is mandatory to prevent possible auditory complications.

\section{REFERENCES}

[1] A. Gkoltsiouk, M. Tzoufi, S. Counsell, M. Rutherford and F. Cowan, "Serial Brain MRI and Ultrasound Findings: Relation to Gestational Age, Bilirubin Level, Neonatal Neurologic Status, and Neurodevelopmental Outcome in Infants at Risk of Kernicterus," Early Human Development, Vol. 84, No. 12, 2008, pp. 829-838.

doi:10.1016/j.earlhumdev.2008.09.008

[2] N. Y. Boo, A. S. Rohani and A. Asma, "Detection of Sensorineural Hearing Loss Using Automated Auditory Brainstem-Evoked Response and Transient-Evoked Otoacoustic Emissions in at Term Neonates with Severe Hyperbilirubinemia," Singapore Medical Journal, Vol. 49, No. 3, 2008, pp. 209-214.

[3] S. Garza-Morales, A. Poblano, A. Robledo-Galván and L. A. Fernández-Carrocera, "Auditory Evoked Potentials in Children at Neonatal Risk for Hypoacusis," Panamerican Journal of Public Health, Vol. 2, No. 4, 1997, pp. 232237. doi:10.1590/S1020-49891997001000002

[4] S. B. Amin, C. Ahlfors, M. S. Orlando, L. E. Dalzell, K. S. Merle and R. Guillet, "Bilirubin and Serial Auditory Brainstem Responses in Premature Infants," Pediatrics, 
Vol. 107, No. 4, 2001, pp. 664-670. doi:10.1542/peds.107.4.664

[5] Y. Yilmaz, S. Degirmenci, F. Akdas, S. Kulekci, A. Ciprut, S. Yuksel, F. Yildiz, L. Karadeniz and A. Say, "Prognostic Value of Auditory Brainstem Response for Neurologic Outcome in Patients with Neonatal Indirect Hyperbilirubinemia," Journal of Child Neurology, Vol. 16, No. 10, 2001, pp. 772-775. doi: $10.1177 / 088307380101601014$

[6] P. Sharma, N. P. Chhangani, K. R. Meena, R. Jora, N. Sharma and B. D. Gupta, "Brainstem Evoked Response Audiometry (BAER) in Neonates with Hyperbilirubinemia," Indian Journal of Pediatrics, Vol. 73, No. 5, 2006, pp. 413-416. doi:10.1007/BF02758564

[7] C. Oysu, I. Aslan, A. Ulubil and N. Baserer, "Incidence of Cochlear Involvement in Hyperbilirubinemic Deafness," Annals of Otology, Rhinology and Laryngology, Vol. 111, No. 11, 2002, pp. 1021-1025.

[8] A. Poblano, I. Chayo, J. Ibarra and E. Rueda, "Electrophysiological and Behavioral Methods in Early Detection of Hearing Impairment," Archives of Medical Research, Vol. 31, No. 1, 2000, pp. 75-80. doi:10.1016/S0188-4409(99)00060-0

[9] C. L. Pereira, A. M. Aguilar, F. Rivera, L. Cruz, I. Santos and A. Poblano, "Concordance between Neurologic Screening Test and Neurologic Examination in Newborns," Clinical Pediatrics (Philadelphia), Vol. 45, No. 4, 2006, pp. 315-323.

[10] S. P. Harrison and I. M. Barlow, "Three Direct Spectrophotometric Methods for Determination of Total Bilirubin in Neonatal and Adult Serum, Adapted to the Technicon RA-1000 Analyzer," Clinical Chemistry, Vol. 35, No. 9, 1989, pp. 1980-1986.

[11] J. How and M. E. Lutman, "Transient Evoked Otoacoustic Emissions Input-Output Function Variation in a Large Sample of Neonates and Implications for Hearing Screening," International Journal of Audiology, Vol. 46, No. 11, 2007, pp. 670-679. doi:10.1080/14992020701438813

[12] M. R. Nuwer, M. Aminoff, D. Goodin, S. Matsuoka, F Mauguiere, A. Star A and J. F. Vibert, "IFCN Recommended Standards from Brain-Stem Auditory Evoked Potentials. Report of IFCN Committee," Electroencephalography and Clinical Neurophysiology, Vol. 91, No. 1, 1994, pp. 112-117. doi:10.1016/0013-4694(94)90013-2

[13] A. Poblano, A. Belmont, J. Sosa, J. Ibarra, Y. Rosas, V. López and S. Garza, "Effects of Prenatal Exposure to Carbamazepine on Brainstem Auditory Evoked Potentials in Infants of Epileptic Mothers," Journal of Child Neurology, Vol. 17, No. 5, 2002, pp. 364-368. doi: $10.1177 / 088307380201700510$

[14] A. Poblano, A. Belmont, J. Sosa, J. Ibarra, A. M. Vargas,
G. Limón and C. Martínez, "Amikacin Alters Auditory Brainstem Conduction Time in Newborns," Journal of Perinatal Medicine, Vol. 31, No. 3, 2003, pp. 237-241. doi:10.1515/JPM.2003.032

[15] M. Ferrán-Aranaz, "Statistical Analyses in SPSS for Windows," Osbourne-McGraw Hill, Madrid, 2001, pp. 19-28.

[16] M. F. Notter and J. W. Kending, "Differential Sensitivity of Neural Cells to Bilirubin Toxicity," Experimental Neurology, Vol. 94, No. 3, 1986, pp. 670-682. doi:10.1016/0014-4886(86)90246-3

[17] A. S. Falcao, C. Bellarosa, A. Fernández, M. A. Brito, R. F. Silva, C. Tiribelli and D. Brites, "Role of Multidrug Ressistence-Associated Protein 1 Expression in the in $\mathrm{Vi}$ tro Susceptibility of Rat Nerve Cell to Unconjugated Bilirubin," Neuroscience, Vol. 144, No. 3, 2007, pp. 878-888. doi:10.1016/j.neuroscience.2006.10.026

[18] P. Govaert, M. Lequin, R. Swarte, S. Robben, R. de Coo, N. Weisglas-Kuperus, Y. de Rijke, M. Sinassappel and J. Barkovich, "Changes in Globus Pallidus with (pre) Term Kernicterus," Pediatrics, Vol. 112, No. 6, 2003, pp. 12561263. doi:10.1542/peds.112.6.1256

[19] M. C. Harris, J. C. Bernbaum, J. Polin, R. Zimmerman and R. Polin, "Developmental Follow-Up of Breastfed Term and Near-Term Infants with Marked Hyperbilirubinemia," Pediatrics, Vol. 107, No. 5, 2001, pp. 10751080. doi: $10.1542 /$ peds. 107.5 .1075

[20] C. E. Ahlfors, S. B. Amin and A. E. Parker, "Unbound Bilirubin Predicts Abnormal Automated Auditory Brainstem Responses in a Diverse Newborns Population," Journal of Perinatology, Vol. 29, No. 4, 2009, pp. 305-309. doi:10.1038/jp.2008.199

[21] C. G. Nwaesei, J. van Aerde, M. Boyden and M. Peerlman, "Changes in Auditory Brainstem Responses in Hyperbilirubinemic Infants before and after Exchange Transfusion," Pediatrics, Vol. 74, No. 5, 1984, pp. 800-803.

[22] K. A. Jangaard, D. B. Fell, L. Doods and A. C. Allen, "Outcomes in a Population of Healthy Term and NearTerm Infants with Serum Bilirubin Levels of $\geq 325$ $\mu \mathrm{mol} / \mathrm{L}(\geq 19 \mathrm{mg} / \mathrm{dL})$ Who Were Born in Nova Scotia, Canada, between 1994 and 2000," Pediatrics, Vol. 122, No. 1, 2008, pp. 119-124. doi:10.1542/peds.2007-0967

[23] K. Xionis, Y. Weirather, H. Mavoori, S. H. Shaha and L. M. Iwamoto, "Extremely Low Birth Weight Infants Are at High Risk for Auditory Neuropathy," Journal of Perinatology, Vol. 27, No. 11, 2007, pp. 718-723. doi: $10.1038 /$ sj.jp. 7211803

[24] S. B. Amin, D. Prinzing and G. Myers, "Hyperbilirubinemia and Language Delay in Premature Infants," Pediatrics, Vol. 123, No. 1, 2009, pp. 327-321. doi:10.1542/peds.2007-3723 